\title{
EL TRABAJO INDEPENDIENTE COMO MÉTODO DE ENSEÑANZA EN LA CLASE ENCUENTRO. UNA EXPERIENCIA PEDAGÓGICA DE LA DISCIPLINA FORMACIÓN PEDAGÓGICA GENERAL
}

\author{
Independent Work as a Teaching Method in the encounter class. A Pedagogical \\ Experience of the Discipline General Pedagogical Training
}

\author{
Omar Elias Aulet Álvarez, Dr. C \\ Universidad de Oriente, Cuba \\ https://orcid.org/0000-0001-8031-0823 \\ oaulet@uo.edu.cu
}

\author{
Mirta Felicita Mayet Wilson, Dra. C. \\ Universidad de Oriente, Cuba \\ https://orcid.org/0000-0003-1384-5355 \\ mirta@uo.edu.cu
}

\author{
Arnaldo Delgado Luque, MsC. \\ Universidad de Oriente, Cuba \\ https://orcid.org/0000-0002-1565-9233 \\ arnaldod@uo.edu.cu
}

Palabras claves: Trabajo Independiente, Método de Enseñanza, Clase Encuentro, Actividades Metodológicas, Clase Modelo, Gestión del Conocimiento, Docentes, Estudiantes.

Keywords: Work Independent, Teaching Method, Class Encounter, Methodological Activities, Class Model, Administration of the Knowledge, Educational, Students.

\author{
Recibido: 01 de octubre de 2020
}

Aceptado: 22 de diciembre de 2020

\section{RESUMEN}

El objetivo es la sistematización teórico-práctica del trabajo independiente como método de enseñanza en la clase, a través del trabajo metodológico integrado en la Disciplina Formación Pedagógica General, para carreras pedagógicas. El uso de los métodos investigativos: análisis-síntesis y el sistémico-estructural, permitió la fundamentación de las categorías abordadas, en función de garantizar la calidad del aprendizaje desde la gestión del conocimiento de los estudiantes universitarios, se revelaron como limitaciones: la carencia en los estudiantes de recursos pedagógicos para enfrentar con independencia cognoscitiva la gestión de sus propios aprendizajes, dificultades en los docentes para el diseño, orientación y control del trabajo independiente. Como resultado se ejemplifica con un conjunto de clases de las asignaturas de la disciplina FPG, cuyo impacto generó cambios positivos en el $90 \%$ de los docentes al asumir el trabajo independiente como método de enseñanza fundamental. Basado en este estudio una de los resultados mejor logrado fue la impartición de un curso de postgrado a los docentes universitarios de otras carreras y facultades.

\section{ABSTRACT}

The objective of the study resided in theoretical-practical systematizing of the independent work as method of fundamental teaching in the class encounter, that like decencies form in the Cuban Superior Education, through the methodological work integrated in the Discipline General Pedagogic Formation, for pedagogic careers. The use of the investigative methods: analysis-synthesis and the systemic-structural one, allowed the foundation of the approached essential categories, in function of guaranteeing the quality of the learning from the administration of the knowledge on the part of the university students in the pedagogic process, they were revealed as main limitations: the lack in the students of the course for workers, of enough pedagogic resources, to face with cognitive independence the administration of their own learnings, difficulties in the educational ones for the design, orientation and control of the independent work during the class encounter. As a result, it is exemplified with a group of classes of the subjects of the discipline FPG whose impact generated positive changes in $90 \%$ of the educational ones from this pedagogic community when assuming the independent work as method of fundamental teaching for the class encounter. Based on this study one of the achieved better results it was the impartation from a graduate degree course to the educational university students of other careers and abilities. 


\section{INTRODUCCIÓN}

La gestión del aprendizaje constituye en los momentos actuales una de las problemáticas más importantes del proceso formativo desde las carreras pedagógicas en la Educación Superior. La necesidad que los estudiantes de Curso por Encuentros (CPE), aprendan a aprender, apropiándose de herramientas y procederes didácticas que les permitan la asimilación consciente de los contenidos para la formación profesional pedagógica, y que los mismos se conviertan en significativos, para su actuación en la vida y para la vida, es una aspiración planteada por el Ministerio de Educación Superior (MES) al personal dirigente y docente, coincidiendo en la necesidad de perfeccionar constantemente las formas y métodos del proceso de enseñanza-aprendizaje desarrollador.

Como resultado de la revisión bibliográfica y documental, así como la ejecución del trabajo docente metodológico y científico metodológico desarrollado en el Departamento Disciplina Formación Pedagógica General (DDFPG), se han detectado que entre las causas que más inciden en los resultados alcanzados en las diferentes carreras pedagógicas, está la carencia en los estudiantes del CPE, de recursos pedagógicos suficientes, para enfrentar con independencia cognoscitiva la gestión de sus propios aprendizajes, lo que incide además en la desmotivación de estos por la profesión pedagógica, esto se ha constado desde la práctica pedagógica, la experiencia profesional de los docentes, el diagnóstico pedagógico a los estudiantes, los resultados de controles a clases realizados a docentes en esta modalidad de estudios universitarios, expresado en las insuficiencias como:

- Los docentes de la disciplina aún manifiestan algunas dificultades para el diseño, orientación y control del trabajo independiente durante la clase encuentro.

- No siempre es suficiente la adecuada planificación, orientación y control del estudio individual previo de los estudiantes, como núcleo teórico básico para garantizar la calidad del trabajo independiente como método fundamental de la clase encuentro.

De ahí que en la investigación el colectivo de docentes a su cargo se propusiera como objetivo: argumentar el tratamiento metodológico para la utilización del trabajo independiente como método de enseñanza fundamental en la clase encuentro. Aspiración que se fundamenta desde los presupuestos teórico-metodológicos y prácticos, relacionados con la concepción del trabajo independiente como método de enseñanza fundamental en dicha clase.

\section{DESARROLLO}

El método de enseñanza es el medio que utiliza la didáctica para la orientación del proceso de enseñanza-aprendizaje. La característica principal del método de enseñanza consiste en que va dirigida a un objetivo, e incluye las operaciones y acciones para el logro de este, como son: la planificación y sistematización adecuadas. Este es esencialmente pedagógico, se orienta en lo fundamental a potenciar el aprendizaje de los estudiantes y desde este, lo educativo. Su principal característica está en el carácter director en el proceso de enseñanza-aprendizaje, ya que dirige la actividad del docente que enseña, así como la del estudiante que aprende para alcanzar el objetivo planteado.

En la literatura pedagógica, didáctica y de las metodologías particulares existentes, al alcance de los profesores y los estudiantes, aparece una amplia clasificación de los métodos de enseñanza, de acuerdo a disímiles criterios de clasificación, que asumen indistintamente los autores, lo que ha creado algunas polémicas en las conceptualizaciones, estas no se contradicen en lo esencial, sino que permiten ver el proceso desde diferentes ángulos.

Por su parte, el trabajo independiente se ha enfocado como método por algunos, otros lo han considerado como una forma de organización del proceso de enseñanza. A nuestro juicio, uno de los criterios más sólidamente argumentado es el de Pidkasisty, 1985; lo analiza como un medio de inclusión en la actividad cognoscitiva, lógica y psicológica de los estudiantes, mediante un proceso de asimilación consciente lo que presupone que el profesor realice un preciso diseño, orientación, y control de éste en función de los objetivos que se plantean en el modelo del profesional y los de las asignaturas que conforman las diferentes disciplinas.

El trabajo independiente responde a determinadas exigencias entre las cuales se señalan las siguientes:

- Presupone la motivación por la significación de la tarea y por la precisión de los objetivos a lograr.

- Prevé conocimientos teóricos previos en los estudiantes.

- Implica el dominio de métodos y medios de trabajo independiente.

- Posibilita el desarrollo progresivo del pensamiento lógico de los estudiantes.

- Presupone la necesidad de ayuda al estudiante (consultas).

- Exige del uso de los medios técnicos orientados para su realización. 
- Contribuye a la interacción profesor- estudiante y estudiante- estudiante.

- Contribuye a la motivación profesional y a la formación de valores positivos de la personalidad del estudiante.

- Implica creatividad e independencia en la realización de las actividades.

- Contribuye al desarrollo de hábitos y habilidades de autoestudio y de autoevaluación en los estudiantes.

- Exige de una planificación y organización lógica de la actividad docente y de requerimientos psicológicos de los implicados.

En la clase encuentro, el trabajo independiente se asume como método de enseñanza fundamental ya que incentiva al estudiante al autoconocimiento, a la búsqueda constante de información, la investigación, promueve a la concientización del mismo a la gestión del conocimiento, como cualidad necesaria que tiene que distinguir al estudiante de la educación superior en las condiciones actuales del nuevo plan de estudio y el perfeccionamiento educacional que se acomete.

La utilización del trabajo independiente como método de enseñanza fundamental en esta forma de docencia, implica concebirlo como un proceso de construcción y reconstrucción por parte del docente y los estudiantes de los conocimientos, habilidades, actitudes, valores en vínculo con los contenidos que se abordan. Permite desarrollar una lógica en el tratamiento a los contenidos seleccionados, que dirige el docente que enseña, pero que tiene que ser protagónico el estudiante que aprende. Se asume como método de enseñanza fundamental en la clase encuentro por las razones siguientes:

- Está presente en todos los momentos de la clase encuentro: el antes, el durante y el después de la actividad docente.

- Es la vía más certera de contribuir a través de esta forma de docencia a la autogestión del conocimiento en los estudiantes como exigencia de la Educación Superior.

- Como método de enseñanza, por su carácter, dirige tanto la actividad del docente en la gestión por enseñar, como la de los estudiantes en la gestión por aprender.

- Adquiere una objetividad y lógica pedagógica, que se aplica a través de los procedimientos que lo apoyan, presente en todas las fases de esta forma de docencia.

- Logra la total dirección pedagógica colectiva del proceso de enseñanza-aprendizaje, en las manos del docente que enseña y los estudiantes que aprenden.

- Se apoya en un sistema de tareas docentes que, por su concreción e interrelación con lo académico, lo laboral y lo investigativo, contribuye al desarrollo de los procesos del pensamiento lógico, por lo tanto, incluye el aspecto procesal, el operacional y los procesos meta-cognitivos, o sea, la reflexión y la regulación metacognitiva, bajo la dirección del profesor que enseña y el estudiante que aprende.

El objetivo del trabajo independiente como método fundamental en la clase encuentro, consiste en garantizar la dirección didáctica para la gestión del conocimiento tanto del docente que enseña como del estudiante que aprende en el proceso pedagógico. En este tipo de clase, ha de transitar por las siguientes fases o etapas para su utilización:

1. Planificación del método: En este momento inicial, de trabajo de mesa, el docente una vez ya concebido el objetivo de su clase, seleccionado el contenido a tratar, y sobre la base del diagnóstico pedagógico de los estudiantes del grupo concibe la utilización del método y los procedimientos a emplear durante las actividades docentes a desarrollar en cada uno de los momentos de su clase encuentro.

2. Ejecución del método: Durante todos los momentos de la clase, es decir, la introducción, el desarrollo y las conclusiones, del docente y los estudiantes ejecutarán las acciones que evidencian la interacción entre ambos y de esta forma se garantiza el autoconocimiento y la autogestión en la búsqueda y procesamiento de la información y con ello la apropiación de saberes en los estudiantes.

3. Control y orientación del método: El docente desde la fase de planificación ya concibe en el momento de la clase en que va a realizar el control del trabajo independiente, teniendo en cuenta todos los requerimientos metodológicos para dicho control y evaluación del mismo. Así como la orientación efectiva del mismo a través de la guía de estudio para la próxima actividad docente.

El trabajo independiente como método de enseñanza fundamental en la clase encuentro, incluye un sistema de procedimientos didácticos dirigidos a:

- La asimilación consciente del material docente.

- El perfeccionamiento de los conocimientos y su desarrollo.

- La consolidación de los conocimientos.

- La formación de habilidades prácticas y profesionales.

- La tendencia a la búsqueda independiente de nuevos conocimientos. 
- Promover las potencialidades individuales de los estudiantes.

- Educación de las cualidades morales y de educación formal positivas en la personalidad de los estudiantes.

Constituyen procedimientos didácticos para dicho método, entre otros los siguientes: el diálogo, la explicación, el debate, la lectura comentada, la ejemplificación, el análisis, la síntesis, el resumen, el subrayado de ideas esenciales, uso de técnicas participativas, uso de fichas de contenido, uso de mapas conceptuales, gráficos, tablas, etc.

La clase encuentro muestra actualmente una tendencia predominante en las carreras universitarias, en los centros universitarios municipales, de conjunto con la educación a distancia y la educación en línea; aunque también está presente en las sedes centrales, unidades docentes y en aulas y áreas propias de organismos, organizaciones y entidades laborales afines con los que las universidades tienen convenios a estos efectos.

El curso por encuentros corresponde a la modalidad semipresencial, la cual se caracteriza por concebir el desarrollo del contenido previsto para la disciplina o asignatura con una carga horaria lectiva menor en comparación con la modalidad presencial y, en consecuencia, también con menos cantidad de actividades académicas que requieran la presencia física simultánea de estudiantes, profesores y personal docente en general; lo que a su vez determina las especificidades de la actividad docente en ese tipo de curso.

La clase encuentro es el tipo de clase típica y más generalizada para desarrollar el trabajo docente presencial de las asignaturas en este tipo de curso, y posee tres estructuras diferentes, según se trate: la clase encuentro inicial o introductoria, las clases encuentro intermedias y la clase encuentro final o integradora. En todas se deben cumplir los requisitos siguientes:

- Presentar los núcleos esenciales del contenido de forma lógica y pedagógica, identificando su vínculo con los conocimientos precedentes y subsecuentes.

- Establecer la debida relación del contenido específico con la futura actividad del profesional.

- Ofrecer una orientación detallada de método de enseñanza y los procedimientos didácticos a seguir en las actividades docentes planificadas, apoyados en las guías de estudio de la asignatura.

Los resultados de la investigación se han introducido en diez facultades, veinte y seis carreras, un total de 1238 estudiantes que transitan por esta forma de docencia, desde el curso escolar 2016-2018 hasta el presente. Se imparte actualmente un curso de postgrado, sobre el tema como parte de la superación postgraduada a 23 docentes universitarios de la carrera Educación Informática, en la Facultad de Ingeniería en Telecomunicaciones e Informática y Biomédica, con una excelente aceptación, lo que da cuenta de su impacto en la formación profesional de los docentes y egresados universitarios. A continuación, y en el orden de lo práctico, se muestran dos de clases modelos presentadas y analizadas a través del sistema de trabajo metodológico integrado que se realiza en el Departamento Disciplina FPG.

\section{Ejemplificación con dos clases de las asignaturas de la disciplina FPG.}

\section{Clase modelo: Psicología II}

La clase pertenece a la asignatura Psicología II, programa que se imparte en el primer o segundo semestre del 1er o 2do año del curso por encuentro, Plan de Estudio E, en todas las carreras pedagógicas, en el caso de la formación de profesores para la educación media y media superior cuenta con un total de 18 horas clases, que se imparten en nueve encuentros.

El objetivo general del Programa de Psicología II están dirigidos a: Caracterizar el aprendizaje del adolescente y el joven, a partir del proceso comunicativo en su grupo escolar y desde la perspectiva del rol profesional del maestro.

\section{Actividad Docente $9 / 10$}

Tema 2: La comunicación educativa en el proceso pedagógico.

Sumario: La comunicación en el proceso pedagógico. Modelos comunicativos que sustentan la práctica pedagógica. Relación entre comunicación y personalidad.

Objetivo: Explicar la comunicación en el proceso pedagógico a partir de su definición, destacando los modelos comunicativos que sustentan la práctica pedagógica y la relación entre comunicación y personalidad, teniendo en cuenta la importancia de estos contenidos para la formación profesional del educador.

Invariantes de la habilidad explicar: 
- Argumentar los juicios de partida.

- Establecer las interrelaciones de los argumentos.

- Ordenar de forma lógica las interrelaciones encontradas.

- Exponer ordenadamente los juicios y razonamientos asociados a las relaciones causales referidas al objeto.

Método: Trabajo independiente.

Procedimientos: Conversación, técnicas participativas, análisis y síntesis.

Bibliografía básica

1. Colectivo de autores. Comunicación Educativa. P 1-13

2. Colectivo de autores. Psicología Para Educadores Capítulo 2 P. 66

3. Colectivo de autores. La Personalidad su Diagnóstico y su Desarrollo. P. 182.

4. Bermúdez, Raquel. Comunicación Positiva. (Material en PDF). Pág. 13

5. Colectivo de autores. Comunicación y Educación (Material en PDF).

Bibliografía complementaria

6. Carpeta Materiales y libros donde aparecen: Resúmenes y Power Point realizados por los profesores del colectivo de la asignatura que facilitarán el estudio del tema.

7. Aula virtual de Psicología II

\section{Fase 1 Introducción (Sistematización)}

Organización del grupo para iniciar la actividad docente. Analizar y conocer posibles ausencias y llegadas tarde, realizar el trabajo educativo correspondiente.

Comenzar recordando que en el encuentro anterior se culminó el estudio del tema I. El proceso de aprendizaje en la adolescencia y en la juventud. (Este recordatorio lo podrá realizar uno, varios estudiantes o el profesor). Se hará énfasis en los aspectos fundamentales del tema, como la definición de aprendizaje, los estilos de aprendizaje, tipos y estrategias de aprendizaje, métodos y técnicas para su estudio, la importancia de estos contenidos para el desempeño profesional del educador, se destacará como este contenido es muy importante para la asignatura Didáctica que recibirán en el segundo semestre.

Se analizarán las posibles dificultades o dudas que presentaron los estudiantes en la realización de Guía de estudio N. 5 orientada en la clase anterior y la localización de las fuentes bibliográficas recomendadas.

Este momento es muy importante para que se expresen y aclaren las dudas donde los estudiantes mismos las podrán esclarecer a sus compañeros a partir del estudio realizado.

\section{Motivación}

El hombre nace individuo y deviene personalidad en el proceso de socialización el que le permite insertarse en la sociedad como un ente social. ¿A través de qué proceso el hombre establece relaciones con los demás? Aplicar lluvia de ideas. Se plantearán las siguientes interrogantes.

- ¿Qué es la comunicación?

- ¿Qué relación existe entre comunicación y educación?

- ¿En qué modelos comunicativos se sustenta la práctica pedagógica de la escuela cubana actual?

- ¿Qué relación se puede establecer entre comunicación y personalidad?

Dar a conocer el tema donde se realizará una síntesis de los contenidos fundamentales del tema, orientar hacia el objetivo y dar a conocer el sumario de actividad docente.

Desarrollo (Tratamiento al nuevo contenido)

Se retoma la Guía de Estudio No. 5, que fue orientada en el encuentro anterior. Se anotan en la pizarra los indicadores para la evaluación del trabajo independiente:

- Independencia cognoscitiva

- Calidad de las respuestas (profundidad, elaboración personal, ajuste al tema)

- Coherencia en las expresiones.

- Dicción y uso adecuado del vocabulario técnico de la asignatura. 
Guía de estudio N. 5

Psicología II

Tema 2: La comunicación educativa en el proceso pedagógico.

Sumario: La comunicación en el proceso pedagógico. Modelos comunicativos que sustentan la práctica pedagógica. Relación entre comunicación y personalidad.

Objetivo: Explicar la comunicación en el proceso pedagógico a partir de su definición, destacando los modelos comunicativos que sustentan la práctica pedagógica, teniendo en cuenta la importancia de estos contenidos para la formación profesional del educador.

Bibliografía básica

1. Colectivo de autores. Comunicación Educativa. Pág. 1-13

2. Colectivo de autores. Psicología Para Educadores. Capítulo 2. Pág. 66

3. Colectivo de autores. La Personalidad su Diagnóstico y su Desarrollo. Pág. 182

4. Colectivo de autores. Nociones de Sociología, Psicología y Pedagogía. Pág. 98

5. Bermúdez, Raquel. Comunicación Positiva. Pág. 13

6. Colectivo de autores. Comunicación y Educación Pág. 1-8

7. Ojalvo Victoria. Comunicación educativa, Capítulo 3 Pág. 11

Bibliografía complementaria

8. Carpeta Materiales y libros donde aparecen resúmenes y Power Point realizados por los profesores del colectivo de la asignatura que facilitarán el estudio del tema.

9. Aula virtual de Psicología II.

Actividades para la autopreparación:

El tema de la comunicación es sin lugar a dudas unos de los principales aspectos a considerar en la formación profesional del educador puesto que el proceso educativo y el de enseñanza-aprendizaje son procesos eminentemente comunicativos.

El educador debe ser un excelente comunicador para lo cual necesita comprender los referentes teóricos del mismo. En esta actividad docente conocerás sobre aspectos esenciales para la dirección del Proceso de Enseñanza Aprendizaje, que estudiarás con más profundidad en Didáctica, y sobre la educación que se abordará en Pedagogía.

Lee, analiza y responde:

1. ¿Qué es la comunicación? Para ello analiza la etimología (procedencia, origen) de la palabra. Compara las diferentes definiciones que aparecen en las fuentes bibliográficas recomendadas. Señala los aspectos comunes entre estas e identifica los rasgos esenciales que consideres. A partir de estos elementos elabora tu propia definición.

2. Lee analíticamente en los materiales docentes: Comunicación educativa y Comunicación y Educación, sobre la relación que se establece entre estas categorías. Elabora un resumen que contenga las respuestas a estas interrogantes: ¿Cómo se define a la educación?, ¿Qué es la comunicación educativa? ¿Qué relación existe entre comunicación y educación?

3. A partir de los conocimientos adquiridos en Psicología I sobre personalidad responde ¿Existe relación entre comunicación y personalidad? Argumenta con no menos de tres razones tu respuesta. ¿Cuáles son los tipos de comunicación según Dra. C. Raquel Bermúdez? ¿Qué importancia tiene para la labor profesional del educador estos conocimientos?

Se comenzará analizando la etimología de la palabra comunicación. Se orientará que se copie en la pizarraComunicación es una palabra de origen latino (communicare) que quiere decir compartir o hacer común 0 Comunicación viene del latín communis, común, se refiere al establecimiento de una comunidad con alguien.

Luego se preguntará ¿qué es la comunicación? Se analizarán las diferentes definiciones que lleven los estudiantes. El profesor llevará en tarjetas las definiciones por si los estudiantes no las tuvieran.

- "Un intercambio de pensamientos, sentimientos, emociones."

Los autores cubanos como Raquel Bermúdez y Lorenzo Pérez Martín la definen como: 
- "Proceso de interacción entre los sujetos mediante el cual se producen influencias mutuas que los modifican".

Raquel Bermúdez y Lorenzo Pérez Martín en el libro Comunicación positiva en educación plantean que:

- En Psicología se entiende por comunicación el proceso de interacción sujeto- sujeto, a través del cual se intercambia información, sentimientos, ideas, etc., que produce una influencia mutua que trae como resultado una modificación de ambos.

Según autores del libro Psicología para educadores:

- "Es la expresión más completa de las relaciones humanas donde se produce el intercambio de ideas, actitudes, representaciones y vivencias entre los hombres que constituye un medio esencial de funcionamiento y formación de la personalidad"

Carmen Reinoso Cápiro, Linares Cordero, libro "Nociones de Sociología Psicología Pedagogía,"

- "Comunicación es un proceso en el cual transcurre la interacción entre los sujetos y el intercambio de información, de vivencias e influencias mutuas que siempre dejan una huella que favorecen el cambio en los interlocutores".

El profesor planteará que entre educación y comunicación existe una ineludible relación para analizar esta relación se partirá de la definición de educación, se explicará que esta es una categoría esencial en la asignatura Pedagogía y que ya tuvimos un acercamiento a ella al estudiar la relación entre educación y desarrollo en Psicología I. A partir de la siguiente interrogante dará participación a los estudiantes para que brinden sus opiniones.

- ¿Cómo se define a la educación?

Retomar la definición que se trabajó en Psicología I. Educación: en su sentido más amplio es el conjunto de influencias recíprocas que establecen entre el individuo y la sociedad, con el fin de lograr su inserción plena en ella o sea la socialización del sujeto. Se considera a la educación como un sistema de influencias que tiene como fin el logro de la socialización del individuo a partir de los intereses y valores de una sociedad, en un contexto determinado.

La siguiente pregunta: ¿Qué es la comunicación educativa? se analizará como un área específica de las Ciencias de la Educación y cuya elaboración teórica metodológica no es aún una construcción acabada.

\section{En el área pedagógica se distinguen dos enfoques}

La comunicación educativa instrumental:

Se enfatiza la comunicación como técnica e instrumento valioso para la educación. Se atiende aquí a la didáctica de los medios de enseñanza y el control del sistema de transmisión entre docente y alumnos con vistas al logro de los objetivos propuestos, así como al uso de técnicas comunicativas utilizadas por el profesor como recursos para que el mensaje llegue al alumno mejor. Este enfoque se corresponde con el primer y segundo modelo de educación ya analizados.

La comunicación educativa procesal: En este enfoque los procesos comunicativos no son instrumentos o estrategias de aprendizaje, sino que constituyen su esencia. En este caso se centra la atención en el proceso mismo y no solamente en sus resultados. Se destaca el papel de la interacción, de la elaboración conjunta de significados entre los participantes como característica esencial del proceso pedagógico.

¿Qué relación existe entre comunicación y educación?

Los estudiantes darán diferentes respuestas partiendo en primer lugar que:

> En el desarrollo histórico de la escuela como institución social, los vínculos entre educación y comunicación se han hecho cada vez más explícitos por el carácter participativo e interactivo de ambos procesos.

> En el proceso pedagógico se manifiestan una gama de relaciones interpersonales, que en los variados matices que adopta la comunicación ejerce influencias específicas en el desarrollo de la personalidad de los educandos.

Se puntualizará que la actividad educativa es una actividad comunicativa por excelencia. La base de la educación es precisamente la comunicación. A través de la comunicación se brinda la enseñanza y a su vez se ejerce una influencia educativa sobre el escolar en un medio participativo. 
Concluida esta actividad se evaluará a los participantes teniendo en cuenta los indicadores señalados. Se evaluará cualitativamente, se llevará un registro de las participaciones y de la calidad de las mismas. Se les pedirá a los alumnos que expliquen las relaciones que se establecen entre los modelos educativos y comunicativos para lo que expondrán el resumen realizado en forma de esquema.

Los estudiantes compararán los modelos destacando que cada uno es superior al anterior, pero a su vez cada cual es el resultado de las condiciones socio-históricas en que surgió y constituyen eslabones valiosos del desarrollo de las ciencias pedagógicas, con aportes importantes para elevar la calidad de la práctica.

A la pregunta de: valora a partir de esta comparación cuál consideras debe ser el modelo que sustenta la práctica pedagógica en la escuela cubana actual ¿por qué? Los estudiantes explicarán que la práctica escolar contemporánea está matizada por la interpenetración de estos tres modelos de acuerdo a las condiciones concretas en que transcurra el proceso pedagógico, es decir coexisten estos tres modelos.

Al responder sobre los tipos de comunicación los estudiantes explicarán que aun cuando cada proceso comunicativo es único e irrepetible en su individualidad concreta, ya que ni siquiera dos momentos diferentes de la comunicación entre las mismas personas son exactamente iguales, sin embargo, de un modo $u$ otro, los distintos procesos comunicativos pueden agruparse de algún modo en dos grandes tipos: aquél en que se toma al otro como objeto, y aquél en que se le toma como sujeto.

\section{* Comunicación donde se toma al otro como objeto:}

En este tipo de comunicación, el otro es un medio para alcanzar un fin. La característica de este tipo de comunicación es que uno de los que entra en este proceso trata de utilizar al otro, de manipularlo, para alcanzar sus propios fines, sin que verdaderamente le importe cómo piensa y siente, intentando que se subordine a sus propios intereses. El otro se siente utilizado y reacciona en consecuencia, lo que trae como resultado dificultades en la comunicación.

Poner ejemplos de este tipo de comunicación: cuando el profesor desarrolla el proceso docente-educativo pensando sólo en sus objetivos, en lo que le conviene, trata a sus alumnos como objetos; cuando en una pareja, uno de ellos maneja la relación en función de sus deseos y necesidades, sin tomar en cuenta los del otro, trata a éste como objeto.

Individualmente, este tipo de comunicación menoscaba la dignidad de una de las partes, y es una fuente de conflictos. En sus casos extremos lleva al maltrato psicológico, verbal y hasta físico.

* Comunicación donde se toma al otro como sujeto:

A diferencia del tipo anterior, en éste uno al otro se respeta como personalidad, respetan su dignidad, reconocen sus derechos mutuos y aceptan su individualidad.

Este tipo de comunicación permite establecer relaciones interpersonales adecuadas, por lo que los educadores deben establecerlas y tratar de que sus educandos las establezcan entre sí. Con esto permiten que el proceso educativo se desarrolle de modo satisfactorio y se alcancen los objetivos que, entre todos, se hayan establecido.

Contribuye, de manera decisiva, al crecimiento personal de quienes la establecen, al permitir el intercambio en un plano de igualdad y el enriquecimiento mutuo al brindarse y recibir cada uno desde su individualidad.

Se evaluarán a los participantes atendiendo a los indicadores establecidos y aplicando el auto, hetero y cohevaluación.

\section{Conclusiones}

Se realizarán conclusiones parciales de esta segunda fase de la clase se destacará el logro del objetivo propuesto y la productividad de la actividad teniendo en cuenta la calidad de las participaciones, se estimulará a los estudiantes que hayan tenido buena calidad en su preparación y se exhortará a aquellos que han quedado rezagados a tener una mejor autopreparación.

Tercera fase (Preparación para el próximo encuentro).

A la hora de comunicarse, además de lo visto hasta aquí, es necesario tener en cuenta, toda una serie de factores que, de una manera u otra, influyen en que la comunicación sea efectiva. La actividad educativa es una actividad comunicativa por excelencia de ahí la necesidad de tener en cuenta otros aspectos que estudiaremos en la próxima clase.

Se orientará la Guía de estudio N.6 donde se precisarán los aspectos relacionados con la ubicación de los contenidos en las fuentes bibliográficas, así como se analizará de forma sintética aspectos del contenido. 
Guía de estudio N. 6

Psicología II

Tema 2: La comunicación educativa en el proceso pedagógico.

Sumario: El lenguaje verbal y no verbal en la comunicación. Su importancia en el proceso pedagógico. Funciones de la comunicación.

Objetivo: Argumentar el papel del lenguaje verbal y no verbal, así como las funciones de la comunicación en el proceso de enseñanza aprendizaje, destacando la necesidad del contenido para la labor profesional del educador.

Bibliografía básica:

1. Bermúdez Morris Raquel. Comunicación positiva en educación. Pág. 9

2. Colectivo de autores. Psicología Para Educadores. Pág. 58

3. Colectivo de autores. La personalidad su Diagnóstico y su desarrollo. Pág. 197

4. Ana María Fernández. Comunicación Educativa.

5. Ana María González Soca y Carmen Reynoso Cápiro. Nociones de Sociología Psicología Pedagogía. Pág. 98.

6. González Rey, F. Comunicación educativa. Pág. 1-9.

7. Ojalvo Victoria. Comunicación educativa. Capítulo 6. Pág. 4

Bibliografía complementaria:

8. Carpeta Materiales y libros donde aparecen: Resúmenes y PowerPoint realizados por los profesores del colectivo de la asignatura.

9. Aula virtual de Psicología II.

Principales conceptos y categorías que debes dominar: Comunicación, lenguaje verbal, lenguaje no verbal o extra verbal, funciones de la comunicación, barreras de la comunicación.

Actividades para la autopreparación:

Para que se dé el proceso comunicativo es imprescindible el uso del lenguaje, ya sea de forma verbal o a través de los diversos canales de la comunicación. En esta actividad estudiaremos el lenguaje verbal y el no verbal o también denominado extra verbal para lo cual debes distinguir las diferencias entre lenguaje e idioma, los tipos de lenguaje oral y no verbal, y debes tener presente la importancia que tiene para tu formación profesional pedagógica el dominio de estos contenidos. Además, estudiarás las funciones de la comunicación y cómo estas se cumplen en el proceso de enseñanza aprendizaje.

Lee, analiza en los libros y materiales docentes señalados en la bibliografía y responde:

1. ¿Qué es el lenguaje? Analiza diferentes definiciones y señala la que consideras más completa y por qué ¿Es lo mismo lenguaje que idioma? ¿Por qué? ¿Cómo se clasifica el lenguaje?

2. Continúa la lectura de las fuentes señaladas y responde ¿A qué llamamos lenguaje verbal? Elabora un resumen de este contenido, utilizando las técnicas que desees (llaves, esquemas, etc.) y sus tipos.

3. Lee en el libro de Nociones de Sociología...y define ¿Qué es el lenguaje no verbal o extra verbal? ¿Cuáles son los tipos de lenguaje no verbal? Redacta un párrafo donde argumentes la importancia del lenguaje verbal y no verbal en la profesión pedagógica.

4. Analiza en los libros y materiales recomendados las funciones de la comunicación, explica en qué consiste cada una de ellas, ¿cuál de las funciones se cumple por gran parte de los maestros? Argumenta qué ocurre en la comunicación cuando se prioriza una función en detrimento de las restantes

a. Lee la anécdota "Historia de un cuartel" en el cap. 6 del libro de V. Ojalvo, pág. 8, además de reírte con tan simpática historia, responde ¿Qué puede ocurrir con las órdenes o con las orientaciones verbales? Relaciona este aspecto con las funciones de la comunicación en el desempeño profesional del educador.

5. Argumenta la importancia que tiene para el desempeño profesional del maestro el conocimiento de las funciones de la comunicación. Refiérete a tu especialidad.

Clase modelo: Pedagogía

De acuerdo a su estructura es una clase encuentro intermedia. 
La clase encuentro escogida pertenece a la asignatura Pedagogía, el cual se imparte en el primer semestre, correspondiente al 2do año del Curso Por Encuentro (CPE), del Plan E en todas las carreras pedagógicas de la UO, el programa cuenta con un total de 40 horas clases.

Los objetivos generales del programa de Pedagogía están dirigidos a:

- Fundamentar desde los referentes teóricos, metodológicos y prácticos de la Pedagogía, el diseño y la dirección del proceso educativo.

- Modelar actividades del proceso pedagógico que propicien la solución de los problemas identificados en los contextos de actuación profesional del maestro.

- Aplicar los conocimientos de la política educacional en la labor que realiza, que se evidencie en una actitud comprometida, transformadora y de auto perfeccionamiento que favorezca su crecimiento personal y profesional.

El programa de esta asignatura está estructurado en cuatro temas:

Tema 1. El carácter científico de la Pedagogía. 8 h/c

Tema 2. El rol profesional del maestro. $12 \mathrm{~h} / \mathrm{c}$

Tema 3. Los contextos de actuación del maestro. 10 h/c

Tema 4. El diagnóstico pedagógico integral. $10 \mathrm{~h} / \mathrm{c}$

Se ha seleccionado el Tema 1. El carácter científico de la Pedagogía. En este tema inicial del programa se abordan los referentes teóricos que fundamentan a la Pedagogía como ciencia, contenido esencial en la formación profesional pedagógica de todo educador y poder trabajar con los estudiantes el fenómeno educativo en Cuba, Latinoamérica y el mundo.

\section{Sistema de conocimientos del Tema 1.}

La Pedagogía como ciencia. Objeto de estudio, leyes, principios, categorías. La Política Educacional cubana. Documentos rectores de la política educacional. Modelo de Escuela cubana. Fin y objetivos.

Objetivo del Tema 1. Caracterizar la Pedagogía como ciencia, teniendo en cuenta los rasgos que la distinguen, así como su concreción en la Política Educacional cubana.

\section{Clase Encuentro 3}

Tema 1: El carácter científico de la Pedagogía.

Sumario: La Política Educacional Cubana. Fin, principios y objetivos generales de la educación cubana. El Sistema Nacional de Educación. Documentos rectores de la Política Educacional.

Objetivo: Explicar la Política Educacional Cubana, teniendo en cuenta su fin, principios y los objetivos de la educación cubana como elementos esenciales, así como las peculiaridades del Sistema Nacional de Educación y la importancia para la futura labor profesional del maestro.

Invariantes de la habilidad explicar:

- Interpretar el objeto o información.

- Argumentar los juicios de partida.

- Establecer las interrelaciones de los argumentos.

- Exponer ordenadamente los juicios y razonamientos.

Método de enseñanza: Trabajo Independiente. Se escogió este método para en primer lugar ser consecuente con la temática de la CMI, en segundo lugar, por ser este el método que ha permitir explicar los contenidos esenciales, a través de la búsqueda de información, la revisión bibliográfica, el análisis, la reflexión, la construcción de aprendizajes, en un proceso de interacción entre el profesor y los estudiantes.

Procedimientos: Diálogo, explicación, lectura comentada, ejemplificación, análisis, presentación de resumen, subrayado de ideas esenciales, etc.

Medios de enseñanza: Pizarra, tarjetas, diapositivas. 
Bibliografía básica:

- Colectivo de Autores: Pedagogía. Editorial Pueblo y Educación, La Habana, 1981, (Epígrafe 2.3 - 2.7 )

- Gonzáles Soca, A. M y Reinoso Cápiro, C. Nociones de Sociología, Psicología y Pedagogía. Editorial Pueblo y Educación, La Habana, 2002. p.45 - 47 y $262-265$.

- Labarrere, G. y G. V. Pedagogía. 2 parte Teoría de la Educación, cap. 14, $15,16$.

- Antonio Blanco. Selección de lecturas de Filosofía de la Educación. Editorial Pueblo y Educación, La Habana, 2003, Pág. $84-91$.

- Addine, Fernández, F. Didáctica: teoría y práctica. Pág. 21 - 31.

- Colectivo de autores ICCP - MINED: Pedagogía. Pág. 29 - 34.

- Colectivo de autores. Compendio de Pedagogía. Pág. 6 - 9.

- Chávez, Rodríguez J. A. Acercamiento necesario a la Pedagogía General.

- Bermúdez Morris, Raquel y otros. Pedagogía de la Educación Técnica y Profesional. Editorial Pueblo y Educación. La Habana, 2014. Capítulo 1.

- Aulet Álvarez, O. B. Material docente. Glosario de términos psicopedagógicos. En Soporte digital: español e inglés. 2010

- Bibliografía complementaria:

- Colectivo de autores: Reflexiones Teóricos-Prácticas desde las ciencias de la educación. 24-25.

- Colectivo de autores: Pedagogía de la Educación Técnica y Profesional.

- Material Docente: Elaborado por el colectivo de asignatura de Pedagogía.

Introducción (Preparación para la nueva materia)

\section{Fase 2. Sistematización}

El docente organiza el grupo para iniciar la actividad docente. Realiza el control de la asistencia. Conoce las ausencias y analizar posibles causas de las mismas y las llegadas tarde. Realizar el trabajo educativo correspondiente.

Se rememora el contenido del tema 1 estudiado anteriormente, relacionado con la Pedagogía como Ciencia. Su objeto de estudio, leyes, categorías, métodos y principios.

El docente enfatiza en los rasgos esenciales que dan carácter de ciencia a la Pedagogía, la importancia de este contenido para el docente y como mucho de estos rasgos (categorías, métodos y principios), van a fundamentar también el carácter de ciencia de la Didáctica que recibirán en el segundo semestre de este 2do año.

Con ello estamos trabajando en función de la sistematización de los contenidos de las asignaturas de la disciplina, como parte de la línea de trabajo metodológico del Dpto.

A continuación, el docente analiza las posibles dificultades o dudas que presentaron los estudiantes en la realización del trabajo independiente, (Guía de estudio No.1), orientada en la clase anterior y la localización y revisión de las fuentes bibliográficas recomendadas.

Este momento es muy importante para que se expresen y aclaren las dudas, donde los mismos estudiantes las podrán esclarecer a sus compañeros a partir del estudio realizado, y en docente orienta cómo solucionar las dificultades detectadas.

Para motivar:

El docente puede comentar que, desde la segunda mitad del siglo XX, la pedagogía ha venido sufriendo en el mundo, y en particular en América Latina un sensible debilitamiento, en cuanto a su condición de ciencia de la educación, por diferentes razones objetivas y subjetivas.

¿Qué ustedes consideran al respecto?

¿Es la Pedagogía una ciencia? Argumente.

Luego de escuchar algunas reflexiones, el docente refiere que precisamente en la clase hoy abordaremos lo relacionado con la Pedagogía como ciencia, reorienta el objetivo de la guía de estudio realizada, que coincide con el de la clase y escribir el sumario en la pizarra.

Desarrollo

Fase 3. Tratamiento del nuevo contenido. 
Para el docente realizar el control y evaluación del trabajo independiente orientado, se retoma la guía de estudio 1 . El docente pone en pizarra los indicadores para evaluar, ya conocidos por los estudiantes:

- Independencia cognoscitiva.

- Calidad de las respuestas: dominio del contenido, profundidad, elaboración personal, ajuste al tema.

- Dicción y uso adecuado del vocabulario técnico de la asignatura.

Enfatizar como en este momento se da tratamiento al contenido de la asignatura, previsto para esta clase encuentro, a través del trabajo independiente, que direcciona como método de enseñanza fundamental, la actividad docente del profesor que enseña y de los estudiantes que aprenden, en función de cumplir con el objetivo de la guía de estudio, que es el mismo de la clase. De esta manera se atiende el problema conceptual metodológico formulado y el objetivo de la $\mathrm{CMI}$; todo en correspondencia con la línea de trabajo metodológico del Dpto. este es el hilo conductor que debe conducir todo el tratamiento metodológico de la actividad.

En este momento el docente hace el análisis de la Guía de estudio 1. Para corroborar desde la función de dirección, cómo orientar al colectivo pedagógico de la disciplina para el tratamiento metodológico al diseño, orientación y control del trabajo independiente en la clase encuentro.

Tema 1. La pedagogía como ciencia.

Sumario: La Pedagogía como Ciencia. Su objeto de estudio, leyes, categorías, métodos y principios.

Objetivo: Caracterizar la Pedagogía como ciencia teniendo en cuenta su objeto de estudio, leyes, categorías, métodos y principios que la fundamentan, así como la importancia de la misma para la formación profesional pedagógica del maestro.

Bibliografía.

Addine, Fernández, F. Didáctica: teoría y práctica. Pág. 21 - 31 .

Aulet Álvarez, O. B. Material docente. Glosario de términos psicopedagógicos. En Soporte digital: español e inglés. 2010

Colectivo de autores ICCP - MINED: Pedagogía. Pág. 29 - 34.

Colectivo de autores. Compendio de Pedagogía. Pág. 6 - 9.

Colectivo de autores: Reflexiones teóricos-prácticas desde las ciencias de la educación. 24-25.

Colectivo de autores: Pedagogía de la Educación Técnica y Profesional.

Chávez, Rodríguez J. A. Acercamiento necesario a la Pedagogía General.

Orientaciones metodológicas:

Los estudiantes realizarán las tareas docentes siguientes en función de la autogestión del conocimiento.

- Análisis de las ideas de pedagogos relevantes que aportaron a la Pedagogía.

1. Realiza el estudio analítico en el texto de Pedagogía ICCP-MINED, de la página 29 a la 34 y contesta:

* Elabora un cuadro resumen en que tengas en cuenta a los pedagogos universales, latinoamericanos y cubanos, qué aportaron a la Pedagogía. Tratamiento a la Estrategia de Técnicas de estudio de la FCE.

* ¿Qué tuvieron en común los aportes de los pedagogos?

* ¿Qué vigencia tienen en la actualidad dichos aportes? Ejemplifique.

- Análisis del concepto ciencia y su clasificación.

2. Realiza la lectura en el texto Acercamiento necesario a la Pedagogía General, página 20-21 y contesta:

* ¿Cómo define este autor a la ciencia?

* Compara esta definición con la que aparece en el Glosario de Términos Psicopedagógicos que tienes en el aula virtual. Expresa tu valoración personal. Lee en voz alta varias veces la traducción al idioma inglés del vocablo y su definición. Tratamiento a la Estrategia de la Lengua Inglesa.

* Volviendo al texto inicial ¿Por qué las Ciencias de la Educación? Ejemplifica.

* ¿Cuál es la ciencia que estudia específicamente el fenómeno educativo? 
- Determinación de los rasgos que identifican el carácter de ciencia de la Pedagogía y los argumentos que la justifican.

* ¿Cuáles son los rasgos que le dan carácter de ciencia a la Pedagogía?

- Explica lo esencial de cada uno de ellos. Relaciona las categorías, las leyes y los principios. Para el estudio de las leyes debes auxiliarte del texto de Didáctica: teoría y práctica. Pág. 21-31. Para el estudio de los principios debes auxiliarte del texto Compendio de Pedagogía. Pág. 6-9

* Relaciona con cuáles otras ciencias se relaciona la Pedagogía.

* ¿Cuáles son los métodos que utilizan la Pedagogía para penetrar en lo esencial de su objeto de estudio?

* ¿Cuál es la base metodológica en que se fundamenta la Pedagogía como ciencia? Argumenta

- Expresar juicios de valor acerca de la importancia de la pedagogía para la formación profesional pedagógico del maestro.

* ¿Qué importancia tiene la Pedagógica para el trabajo del maestro? Valora. Tratamiento a la Estrategia de Trabajo Político-Ideológico. (Estrategia de formación vocacional y orientación profesional de la FCE).

* ¿Expresa cómo han contribuido estos contenidos a tu crecimiento personal y profesional?

El docente enfatiza que en la medida que los estudiantes intervienen con sus respuestas a las tareas docentes podrán utilizar la pizarra para exponer los contenidos abordados, $u$ otro medio de enseñanza, y el docente irá haciendo las acotaciones necesarias en función de las invariantes de la habilidad del objetivo de la clase (Interpretar el objeto o información-Argumentar los juicios de partida-Establecer las interrelaciones de los argumentos-Exponer ordenadamente los juicios y razonamientos). Esta acción del docente permitirá en lo posible ir sistematizando también los contenidos para la formación profesional pedagógica de las asignaturas de la disciplina, a través de las categorías interdisciplinarias:

Ej. Núcleos conceptuales:

- Objeto de Estudio,

- Categorías,

- Leyes,

- Principios,

- Base Metodológica,

- Relaciones con otras Ciencias

Retomar que estos mismos rasgos que definen a todas las ciencias, ya los estudió en Psicología, ahora en Pedagogía y luego en Didáctica.

El docente puede ir llevando un registro de las participaciones de los estudiantes los cuales evaluará y controlará en el momento de la intervención o al final. Se evaluará en correspondencia con los indicadores presentados inicialmente. El docente puede utilizar la autoevaluación, la heteroevaluación y la coevaluación.

\section{Fase 4. (Preparación para el próximo encuentro)}

El docente refiere que la Pedagogía en su objeto estudia los procesos educativos en los que participa el sujeto; es decir la educación, que por su carácter clasista responde a los intereses de la clase en el poder, por lo que está obligada a trazar una política educacional en correspondencia con el país y El Estado que lo representa; de ahíla necesidad de que cada país establezca su Política Educacional, aspecto que estaremos abordando en la próxima actividad docente:

- Es el trabajo metodológico es una dirección de trabajo fundamental para proveer a los docentes de los recursos y procedimientos didácticos necesarios para asumir el trabajo independiente como método de enseñanza fundamental en la clase encuentro como forma de docencia en la Educación Superior.

- La utilización del trabajo independiente como método de enseñanza fundamental en la clase encuentro, favorece la independencia cognoscitiva, la investigación y la regulación del comportamiento, en función de la autogestión del aprendizaje en la formación de los estudiantes como profesionales de la Educación.

- El material docente ha permitido orientar a los docentes cómo ofrecer tratamiento metodológico al trabajo independiente como método de enseñanza fundamental para trabajar los contenidos para la formación profesional pedagógica de los estudiantes, a través de la clase encuentro, ejemplificando con varias asignaturas de la disciplina FPG. 


\section{CONCLUSIÓN}

Se realizarán conclusiones de esta primera fase de la clase se destacará el logro del objetivo propuesto, y la efectividad de la actividad docente realizada, teniendo en cuenta la calidad de las participaciones de los estudiantes, se estimulará a los estudiantes que hayan tenido buena calidad en su preparación y exposición de los contenidos, se exhortará a aquellos que han quedado rezagados a tener un mejor desempeño durante la clase en dependencia de la autopreparación, condición indispensable para esta forma de docencia.

El docente orientará el trabajo independiente, a través de la Guía de estudio No.2 donde se precisan los aspectos relacionados con la ubicación de los contenidos en las diferentes fuentes bibliográficas, las orientaciones metodológicas para el tratamiento del mismo, a través de las tareas docentes que realizarán los estudiantes. El docente una vez más refiere como en este momento se orienta el contenido previsto para esta clase encuentro, a través del trabajo independiente, que direcciona como método de enseñanza fundamental, la actividad docente del profesor que enseña y de los estudiantes que aprenden, en función de cumplir con el objetivo de la guía de estudio, que es el mismo de la clase encuentro. De esta manera continuamos atendiendo el problema conceptual metodológico formulado y el objetivo de la CMI; todo en correspondencia con la línea de trabajo metodológico del Dpto. este es el hilo conductor que ha conducido todo el tratamiento metodológico de la actividad presentada.

\section{BIBLIOGRAFÍA}

1. Dewey, John. (1961) La ciencia de la educación. Editorial Losada, Buenos Aires. (2), 106

2. Klingberg, Lothar. (1972) Introducción a la Didáctica General. Editorial Pueblo y Educación. La Habana (4), 272

3. Nérici, Imideo. (1982) Sobre el balance de la situación de la teoría del plan de enseñanza. En revista Pädagogische Forschung, No.2

4. Vigotsky, S. L. (1987) Historia del dearrollo de las funciones psíquicas superiores. Editorial Científica Técnica. La Habana (3) 306

5. Batista, I.; Lema, A. y Delgado, E. La Motivación y Orientación Educativa. http://ojs.formacion.edu.ec/index.php/rif/article/view/12 DOI: https://doi.org/10.34070/rif.v1i1 